\title{
Análisis de la enseñanza de la estructura e interacciones de la materia según la física moderna en primero de bachillerato
}

Study of the teaching process about the structure and interactions of matter through modern physics at the second-to-last year of high school

\section{Paula Tuzón Jordi Solbes}

Universitat de València

Resumen: En este artículo presentamos el análisis de cómo es la enseñanza de la estructura e interacciones de la materia en la asignatura de Física y Química de primero de bachillerato. El objetivo es evaluar si estos contenidos se introducen en su versión más actualizada, en la línea de la física moderna. Para ello, se han analizado distintos libros de texto de esta asignatura a través de un cuestionario y se han hecho entrevistas a profesores que la imparten.

Palabras clave: Física moderna, estructura atómica, interacciones, partículas, modelización.

Abstract: In this study we analyze the teaching process regarding matter structure and interactions on Physics and Chemistry subject in the first course of bachillerato (second-tolast year of high school). Our goal is to evaluate if these contents are presented following an updated picture according to modern physics. For that, several text books of this subject have been analyzed through a questionnaire and some interviews with teachers have been made.

Key Words: Modern physics, atomic structure, interactions, particles, modeling.

(Fecha de recepción: junio, 2014, y de aceptación: septiembre, 2014)

DOI: 7203/DCES.28.3599 


\section{Introducción}

Importancia e interés del tema

De qué está hecha la materia y cómo interaccionan sus componentes son preguntas que han marcado el desarrollo histórico de la física y la química en sus distintos niveles de complejidad. Esto se refleja en el currículum de la asignatura de Física y Química en la educación secundaria, tanto en la etapa obligatoria como en el bachillerato, en la que el tema de estructura e interacciones de la materia marca todos los contenidos; de manera directa cuando se habla de estructura atómica y de la naturaleza de las fuerzas (dinámica), y de manera indirecta o como punto de partida para el desarrollo de otros temas como el de los distintos tipos de enlaces químicos, las interacciones gravitatoria y eléctrica o el tema de trabajo y energía.

El siglo XX ha marcado sin duda alguna un antes y un después en el desarrollo de la física respecto al estudio de los componentes elementales de la materia y sus interacciones: desde la fundamentación teórica de la relatividad y la física cuántica a principios de siglo, hasta la propuesta y consolidación del modelo estándar de la física de partículas a mediados de siglo, que predice la existencia de las partículas fundamentales y ofrece una descripción completa de sus interacciones, y también, durante todo el siglo hasta la actualidad, la detección de cada una de las partículas propuestas por el modelo. Con todo, hoy en día se sabe que la materia, sea estable o inestable, está compuesta por doce partículas elementales, quarks y leptones, que interaccionan de cuatro maneras diferentes: electromagnética, gravitatoria, fuerte y débilmente. Teniendo en cuenta estos ingredientes, la física es capaz de explicar y predecir gran cantidad de fenómenos que ocurren tanto a nivel macroscópico como microscópico, ofreciendo así una imagen bastante completa de nuestro Universo. Sin embargo, aunque el desarrollo curricular no especifica hasta cuándo (históricamente) deben presentarse estos contenidos, el tema de estructura e interacciones de la materia se presenta típicamente hasta lo sucedido a principios del siglo XX en todos los cursos de Física y Química de la educación secundaria y el bachillerato. Es decir, en general no se presentan los contenidos actualizados según lo sucedido en la segunda mitad del siglo XX y lo que llevamos del XXI.

Esta omisión del desarrollo de la física en los últimos 70 u 80 años, no responde a ninguna exigencia curricular ni didáctica; aunque siempre se ha considerado la enseñanza de los temas recientes como más difícil que la de los antiguos (Solbes, 1996). Sin embargo, según algunos estudios (Fischler y Lichtfeldt, 1992) esto puede resultar didácticamente contraproducente, ya que el aprendizaje de los modelos atómicos clásicos (e.g. modelo de Bohr) puede dificultar la posterior compresión de conceptos más actualizados, aunque otros lo consideran necesario para el aprendizaje del estudiante, en especial 
de la naturaleza de la ciencia $(\mathrm{NdC})$ (Kalkanis, Hadzidaki, et al., 2003; Petri y Niedderer, 1998; Sinarcas y Solbes, 2013; Solbes, 1996). Por otra parte, la puesta al límite de dichos modelos por parte de los alumnos lleva a cuestiones que no tienen solución excepto a la luz de modelos atómicos actuales (e.g. ¿por qué los protones no se repelen en el núcleo atómico?) con lo que su introducción en el proceso enseñanza-aprendizaje es imprescindible, especialmente en un marco socio-constructivista. Además, se ha demostrado que los contenidos de física moderna están muy presentes los medios de comunicación (Internet, TV, etc) que utiliza el alumnado y esto despierta su interés por la física ya que, por una parte, permiten una mayor contextualización de las ideas que se discuten (relaciones ciencia-tecnología-sociedad (CTS)) y por otra, responden a preguntas de actualidad (Pérez y Solbes, 2006).

El objetivo del presente trabajo es analizar la presencia de conceptos actualizados en el tema de estructura e interacciones de la materia en el contexto de primero de bachillerato. En este curso se presentan de manera más completa los contenidos que se han ido desarrollando en $3^{\circ}$ y $4^{\circ}$ de la ESO, y es precisamente este tema el que vertebra toda la asignatura de Física y Química. Dichos contenidos no son presentados en la Física de segundo de bachillerato (BOE 147, 2008) aunque en el DOCV aparece un único párrafo sobre partículas en el último tema del curso, Física Nuclear (DOCV 5806, 2008). Esta ubicación nos indica que es muy difícil que el tema se imparta. Esto se confirma cuando se analizan las PAU de Física de las universidades valencianas, que nunca plantean cuestiones al respecto, y con las declaraciones de los profesores, que presentaremos a continuación.

Por todo ello nos planteamos analizar qué tipo de enseñanza del tema se realiza y las ideas que se transmiten a los estudiantes sobre el mismo en la Física y Química de primero de bachillerato, mediante un análisis de los contenidos incluidos en libros de texto, así como a partir de entrevistas personales con profesores de centros de secundaria.

Las preguntas que nos hemos hecho son: i) ¿Qué contenidos conceptuales se enseñan sobre física de partículas en primero de bachillerato? ii) A nivel de naturaleza de la ciencia, ¿los modelos clásicos se presentan como verdades cerradas o abren nuevas perspectivas? ¿Se analizan sus límites? iii) A nivel de relaciones CTS, ¿se habla de aplicación o impacto social de la física de partículas?

\section{Marco teórico}

En el tema de estructura e interacciones de la materia tiene una especial importancia la modelización como estrategia de enseñanza-aprendizaje, ya que el ejemplo paradigmático del uso de modelos como introducción de contenidos es precisamente el bloque de modelos atómicos (Solbes, Silvestre, et $a l ., 2010)$. La estrategia de modelización es, desde el punto de vista de didáctica de las ciencias, clave para garantizar un aprendizaje significativo. Dentro del 
marco socio-constructivista, en el que se pretende que el alumno aprenda de la manera más próxima posible a como se realiza la actividad científica, la modelización es un recurso importante puesto que así lo es también en el desarrollo de las investigaciones científicas cotidianas (Acher, Arcà, et al., 2007; Van Driel y Verloop, 2002; Solbes, 2009).

Según los estándares de la U.S. National Science Education (National Research Council, 1996), "Los modelos son esquemas o estructuras provisionales que se corresponden con objetos reales, situaciones, o tipo de situaciones, con un poder explicador. Los modelos ayudan los científicos e ingenieros a entender cómo funcionan las cosas”. Así que son utilizados como explicación de forma aproximada, esquemática y sencilla de fenómenos que, de otra forma, requerirían una descripción complicada. También permite familiarizar los alumnos con los procedimientos de trabajo de los científicos y la NdC, que elaboran modelos para explicar los problemas hasta que surgen dificultades que obligan a cambiarlos (Gil y Solbes, 1993; Kalkanis, Hadzidaki, et al., 2003; Solbes, 1996).

Las analogías y diferencias del modelo con el fenómeno real han de ser fácilmente identificables. Las analogías sirven para establecer las hipótesis que, por su parte, han de ser contrastables. Las diferencias con el fenómeno son consecuencia de la simplificación (el modelo ha de ser más accesible para la investigación que el fenómeno real). El desarrollo del modelo se considera un proceso iterativo y nunca ha de presen- tarse como una verdad cerrada, los contenidos han de ser revisados después de contrastarse con los datos relacionados con el fenómeno. En este proceso, el nuevo modelo siempre aparece de manera constructiva tras la discusión de los conflictos con modelos anteriores y la observación del fenómeno; por ello, si se utiliza la modelización como recurso de enseñanza-aprendizaje, las estrategias de transmisión-recepción no tienen sentido desde el punto de vista didáctico.

Ahora bien, presentar modelos no implica necesariamente que se haga uso de la estrategia de modelización, si dicha presentación se limita a un conjunto de nombres, fechas y datos históricos. El fracaso de la estrategia se acentúa si, además, la presentación de los modelos se hace hasta un determinado momento en la historia de manera poco justificada, ignorando las versiones más actualizadas; así, las últimas versiones presentadas suelen aparecer sesgadas y con informaciones inconexas.

El tema de estructura e interacciones de la materia vertebra, como hemos dicho, toda la asignatura de Física y Química de primero de bachillerato, pero se trata esencialmente en dos grandes bloques temáticos: el de modelos atómicos y el de dinámica o fuerzas. Es en estos dos bloques donde se desarrollan la mayor parte de los contenidos al respecto.

La historia de estos temas (Fritzsch, 1984; Gell-Mann, 1995; Kragh, 2007; Lederman y Teresi, 1996; Sánchez Ron, 1992, 2006; Tipler, 1985; Velasco, 2000), sin retrotraernos a Demócrito 
y Epicuro, se inicia con Dalton, donde se discute la idea de "discretización" de la materia, sigue con Thomson, donde se introduce el concepto de "carga" y átomo como un ente eléctricamente neutro, y Rutherford, donde se habla por primera vez de "núcleo". Ya en el contexto precuántico, aparece el modelo de Bohr, donde básicamente se discute el electrón y su estado energético dentro de este nuevo paradigma; descripción que se completa con ampliaciones a este modelo o comentarios adicionales (e.g. modelo de Sommerfeld). A grandes rasgos, ese es el hilo argumental hasta principios del XX: la materia está compuesta por átomos, que son entidades electromagnéticas compuestas por protones, neutrones y electrones. El papel de la modelización en este proceso se pierde cuando los conceptos aparecen sin ningún tipo de justificación y los modelos se presentan como verdades cerradas que no abren nuevas perspectivas. A este respecto, la introducción del neutrón es clave; primero, porque con los ingredientes que presentan los modelos citados, la aparición de esta partícula no está del todo justificada, y segundo, porque la discusión acerca de su aparición (nuevas perspectivas) es precisamente la que propicia la introducción de modelos más actualizados donde se contemplan otras partículas e interacciones. El neutrón no fue descubierto, de hecho, hasta 1932 y su papel no se entendió por completo hasta la fundamentación del modelo de quarks.

Respecto a interacciones fundamentales o fuerzas; dentro del tema de estructura de la materia (modelos atómicos), si se tiene en cuenta el contexto histórico de finales del XIX, la interacción fundamental es la eléctrica. Está además la gravedad, que no afecta a la estructura del átomo. Respecto a la estrategia de modelización y con los ingredientes de estos modelos clásicos, hay una pregunta obvia que abre nuevas perspectivas: ¿por qué los protones pueden estar tan juntos en el núcleo si tienen la misma carga eléctrica? Esto propiciaría, al igual que la discusión sobre el neutrón, la introducción de modelos renovados, que generalmente no se hace. En el tema de fuerzas propiamente, donde típicamente se discuten situaciones macroscópicas con la fuerza peso, el rozamiento, las tensiones o la elasticidad, la relación de éstas con las fuerzas fundamentales queda a elección del libro o del profesor.

La comprensión del papel del neutrón en el átomo llegó, como hemos dicho, con el modelo de quarks, es decir, con la introducción de nuevas partículas e interacciones; que a su vez responde a la pregunta sobre los protones, cosa que no es posible si sólo se tiene en cuenta la interacción electromagnética. Prácticamente coincidiendo con el descubrimiento del neutrón, a principios del siglo $\mathrm{XX}$ se detectaron gran cantidad de nuevas partículas provenientes de los rayos cósmicos. Los científicos de la época les dieron nombres según el alfabeto griego: delta, pi, sigma, rho, etc. Ante tal proliferación de nuevas partículas, se pensó si no existiría un factor común a todas ellas, un modelo más sencillo que expli- 
case su naturaleza; de la misma manera que, años atrás, ante la gran cantidad de elementos químicos se buscó un modelo más sencillo basado en protones, neutrones y electrones (átomo clásico) que explicase su clasificación y propiedades. Se llegó a la conclusión de que estas nuevas partículas (hadrones) estaban formadas por seis tipos de quarks, y su diferente combinación en grupos de dos o de tres daba lugar a estas partículas con distintas masas y cargas. Entre ellas están el neutrón y el protón, formados por dos quarks down y uno up y por un quark down y dos up respectivamente. Estas nuevas partículas fundamentales, los quarks, tienen carga eléctrica, pero también experimentan otra interacción entre ellos llamada fuerza nuclear fuerte; más fuerte que la electromagnética y que explica, entre otras cosas, que los protones puedan estar unidos en el núcleo atómico. De la misma manera, se entiende entonces el papel de neutrones, que al estar también compuestos por quarks, contribuyen a la estabilidad del núcleo atómico.

A lo largo del siglo XX se han descubierto tanto los quarks como otras partículas nuevas, el muón y el tau, compañeras del electrón, y los tres tipos de neutrinos. Las partículas elementales que no son quarks se llaman leptones y se diferencian de éstos porque no interaccionan fuertemente. En particular, es interesante el descubrimiento del neutrino, que fue propuesto por Pauli en los años 30 como solución a la "energía perdida" en las reacciones nucleares y fue descubierto en el 56. El neutrino debía tener unas propiedades "especiales", como ser muy poco masivo y no interaccionar prácticamente con nada; y es que el neutrino es neutro eléctricamente (de ahí su nombre), como es un leptón tampoco interacciona fuertemente, y sólo siente la fuerza nuclear débil, otra nueva interacción que se da entre algunas partículas subatómicas y que es responsable de reacciones nucleares como la radioactividad. Con todo, el modelo estándar de la física de partículas completa la visión clásica de estructura e interacciones de la materia con dos interacciones nuevas, la nuclear fuerte y débil, y una "tabla periódica" fundamental con seis leptones y seis quarks, de los cuales, sólo dos quarks y un leptón (el electrón) forman lo que llamamos "materia estable" o átomo. Las interacciones o fuerzas se conciben como intercambio de partículas, a las que se llama bosones, cuyas masas y propiedades explican el alcance y las características de la interacción correspondiente. Esta última reflexión completa la visión del fotón o "cuanto de energía" como responsable de la interacción electromagnética; así, los bosones $\mathrm{W}$ y Z son los mediadores de la interacción débil y los gluones de la fuerte. Para la interacción gravitatoria se suponen los gravitones como mediadores, pero esta es una de las piezas que el modelo estándar todavía no ha incluido en su puzle, y no está de más sacarlo a colación, como uno de los problemas abiertos, atendiendo a la naturaleza de la ciencia, que propone modelos donde existen puntos flacos y respuestas tentativas que todavía no se han comprobado. 
Hay otros conceptos que el siglo XX y el modelo estándar ofrecen y merece la pena que se discutan. Desde el papel o necesidad del bosón de Higgs, recientemente descubierto, a la antimateria y sus aplicaciones (e.g. técnicas de diagnóstico y tratamiento de tumores); así como otras muchas aplicaciones que tiene en la actualidad el desarrollo de la física de partículas (e.g. en informática o tecnología de materiales).

En didáctica de las ciencias, una de las tres vertientes metodológicas a favor de la introducción de toda esta nueva física (Moreira y Ostermann, 2000), es a través de la estrategia de modelización, tal y como hemos discutido al principio (Gil, Senent Pérez, et al., 1987; Gil y Solbes, 1993; Solbes, Calatayud, et al., 1987a). Además de esta, y también como hemos mencionado, existe la vertiente que propone la introducción de nuevos modelos en lugar de los antiguos, es decir, no desde la modelización sino directamente presentando las últimas versiones (Fischler y Lichtfeldt, 1992). Esto está motivado por el argumento de que la introducción previa de los modelos clásicos puede dificultar más que ayudar a la comprensión de los nuevos. Por ejemplo, según estos estudios, resulta difícil abandonar el modelo planetario de Rutherford por la idea de orbital. Finalmente, la última vertiente metodológica propone la selección de algunos temas esenciales de física moderna y que, de esta manera, se ofrezca a los alumnos una "percepción" del panorama moderno (Arons, 1994). Así pues, la literatura muestra ciertas discrepancias en cómo enseñar la nueva física según la importancia que se le dé a los prerrequisitos, al contexto lógico o histórico, o al papel de las analogías clásicas. Respecto a qué enseñar, se observa una concentración en los tópicos de relatividad, partículas elementales y física cuántica. De lejos, el tema de los contenidos es el más discutido, por encima de aspectos metodológicos, epistemológicos, históricos, curriculares y propuestas evaluadas en el aula. Hay estudios también sobre las concepciones alternativas de los estudiantes, generalmente asociadas a errores en los libros de texto o informaciones provenientes de los medios de comunicación, internet, la literatura o el cine (Solbes, Calatayud, et al., 1987b). Finalmente, algunos estudios sobre propuestas llevadas al aula sugieren un éxito en la introducción de temas de física moderna (Cuppari, Rinaudo, et al., 1997; Solbes, Calatayud, et al., 1987a; Stefanel, 1999). Hay áreas importantes de la física moderna que no aparecen discutidas en toda esta literatura, y el tema de formación de profesores y preparación de materiales para enseñar estos contenidos está en general poco tratado (Moreira y Ostermann, 2000; Ostermann y Cavalcanti, 1999).

Con esta situación sobre la mesa, las hipótesis que hacemos frente a nuestras preguntas son que i) los conocimientos sobre física de partículas serán muy limitados y presumiblemente confusos, ii) los modelos se limitan a las versiones clásicas o precuánticas de principios del siglo XX sin mostrar sus limitaciones y sin asomarse a los nuevos modelos de la física de partículas, y iii) no se valora el 
impacto social ni los claros avances en este tema.

\section{Métodos}

Para contrastar las hipótesis formuladas se ha elaborado un cuestionario dirigido a los libros de texto que utilizan los alumnos en la asignatura de Física y Química de primero de bachillerato.Además, este análisis se ha completado con entrevistas a profesores de Física y Química de primero de bachillerato sobre la presencia de conceptos relacionados con física de partículas en las asignaturas que imparten. A continuación, describimos el diseño del cuestionario para libros y cuáles han sido las preguntas que les hemos hecho a los profesores.

Dado que tan sólo en segundo de bachillerato aparece explícitamente en el DOCV (DOCV 5806, 2008) un ítem sobre física de partículas, es de esperar que en los libros de texto de primero no haya un tema cerrado que dé una visión completa del modelo estándar. Aún así, tal y como hemos dicho en la introducción, los temas relacionados con estructura e interacciones de la materia que vertebran todo el currículum de esta asignatura se pueden cubrir con los modelos más actuales y, siguiendo el objetivo de este estudio, lo que queremos evaluar es si los libros de texto de los que normalmente disponen los centros van en esta dirección. Para ello, se ha elaborado un cuestionario (que se puede ver en la Tabla 2 de los resultados) para los siguientes 11 libros de Física y Química de editoriales con gran difusión en nues- tro país y de años recientes (a partir del 2000): Akal, Bruño, Ecir 02, Ecir 08, Edelvives, Editex, McGrawHill, Oxford, Santillana 00, Santillana 09 y SM. Cuando hay dos libros de una misma editorial se distinguen por el año de publicación.

Como se puede ver en la Tabla 2, las preguntas hacen referencia a cómo de actualizados se presentan los conceptos, si están o no en la línea de la visión actual que da la física de partículas. Primero, en las preguntas 1-6, se revisa cómo es la introducción que se hace de estructura e interacciones de la materia y cómo de lejos llegan los modelos presentados, si los modelos clásicos se presentan de manera coherente y si se introducen nuevas perspectivas. En las preguntas 7-10 se tratan cuestiones directamente relacionadas con la física moderna que no necesariamente aparecen de manera natural a partir de los modelos clásicos. En las preguntas 11-13 se cuestiona cuál es la utilización del contexto en la presentación de los modelos y las relaciones CTS. Finalmente, la última pregunta incide en el uso de los nuevos modelos como solución ante los límites de los modelos clásicos.

Se ha realizado un análisis cualitativo de las 14 preguntas del cuestionario. Para resumir los resultados de este análisis y dado que las respuestas son fácilmente clasificables en "sí" o "no", se han contabilizado también las frecuencias de "sí" para los distintos libros de texto. El análisis de los resultados se realizó independientemente por dos investigadores con objeto de contrastar su validez 
Esta revisión de libros se ha completado con entrevistas a 7 profesores de secundaria que se han realizado en el mes de diciembre de 2013. Las entrevistas se han hecho individualmente cubriendo las siguientes preguntas mostradas en la Tabla 1.

\section{Tabla 1. Preguntas para la entrevista con profesores de Física y Química de primero de bachillerato}

1. ¿Se enseña algún concepto, experimento o cuestión CTS relacionada con la física de partículas?

2. En la física de segundo de bachillerato, la física de partículas está contemplada en el currículum. Si has contestado antes que no, ¿podrías explicar por qué no se enseña en segundo?

3. ¿Qué conceptos, experimentos o cuestiones CTS relacionados con la física de partículas se podrían enseñar y en qué cursos?

4. ¿Piensas que su enseñanza puede ayudar a entender mejor la naturaleza de la ciencia, cómo trabajan los científicos? ¿Por qué?

5. ¿Piensas que su enseñanza puede ayudar a la comprensión de las relaciones CTS? ¿Por qué?

6. ¿Piensas que su enseñanza puede mejorar el interés de los alumnos? ¿Por qué?
Con estas preguntas, la entrevista pretende dar una idea de, primero, si se habla o no de física de partículas en primero de bachillerato, sea a través de conceptos, experimentos o relaciones CTS. Se cuestiona también si se hace en segundo de bachillerato, donde el pretexto del currículum no está. Después, se pregunta por los conceptos que el profesor cree que se pueden enseñar. Y finalmente, cuál piensa el profesor que puede ser el impacto de enseñar este tipo de física para que el alumno entienda mejor la naturaleza de la ciencia, las relaciones CTS y/o para mejorar su interés por la asignatura.

\section{Resultados y discusión}

La Tabla 2 resume los resultados del cuestionario para los libros de texto analizados. Las respuestas son en general dicotómicas; nos podemos hacer una idea, por tanto, de la presencia de conceptos renovados en cuanto a estructura e interacciones de la materia haciendo un conteo de los síes y los noes en las respuestas (aunque haya matices que discutir). El resultado global es negativo, ya que sólo un $28.6 \%$ de las respuestas han sido positivas. Las preguntas que han obtenido un número mayor de respuestas positivas son, primero, la pregunta 12 , sobre la utilización de la historia de la ciencia. Todos los libros recurren al contexto histórico en la introducción de los modelos atómicos, y la mayoría lo hacen también en la introducción de las interacciones eléctrica y gravitatoria; este recurso, sin embargo, no aparece dentro 
de una estrategia de modelización en la que se hace referencia al contexto para motivar la construcción de un modelo, sino que consiste más bien en un conjunto de nombres y fechas. Los modelos, además, son clásicos y el recurso histórico es

\section{Tabla 2. Resumen de los resultados positivos del cuestionario pasado a los libros de texto}

\begin{tabular}{l|c|}
\hline Pregunta & $\begin{array}{c}\text { Libros } \\
\text { (N=11) }\end{array}$ \\
\hline $\begin{array}{l}\text { 1.- ¿Aparece alguna mención sobre la existencia de cuatro interacciones } \\
\text { fundamentales? }\end{array}$ & 7 \\
\hline \begin{tabular}{l} 
2.- ¿Se explica con ellas el comportamiento de la materia macroscópica? \\
\hline $\begin{array}{l}\text { 3.- ¿Aparece alguna mención a la necesidad de la interacción fuerte } \\
\text { para explicar la estabilidad del núcleo? }\end{array}$
\end{tabular} & 4 \\
\hline $\begin{array}{l}\text { 4.- ¿Aparece alguna mención a la necesidad de las interacciones débiles } \\
\text { para explicar la radioactividad? }\end{array}$ & 4 \\
\hline $\begin{array}{l}\text { 5.- ¿Aparece alguna mención al fotón no sólo como cuanto del campo } \\
\text { electromagnético, sino como mediador de esta interacción? }\end{array}$ & 2 \\
\hline \begin{tabular}{l} 
6.- ¿Aparece alguna mención a la interacción como intercambio de partículas? \\
\hline 7.- ¿Aparece alguna mención a los aceleradores de partículas?
\end{tabular} & 3 \\
\hline $\begin{array}{l}\text { 8.- ¿Aparece alguna mención sobre el papel de las colisiones en el } \\
\text { estudio de las partículas? }\end{array}$ & 1 \\
\hline $\begin{array}{l}\text { 9.- ¿Aparece alguna mención sobre el papel de los principios de } \\
\text { conservación estudiados (p, E, q) en el estudio de las partículas? }\end{array}$ & 0 \\
\hline $\begin{array}{l}\text { 10.- ¿Aparece alguna mención sobre el papel de los campos eléctricos y } \\
\text { magnéticos en el funcionamiento del acelerador? }\end{array}$ & 11 \\
\hline $\begin{array}{l}\text { 11.- ¿Aparece alguna mención sobre las relaciones CTS en el estudio de } \\
\text { partículas e interacciones? }\end{array}$ & 0 \\
\hline $\begin{array}{l}\text { 12.- ¿Aparece alguna utilización de la historia de la ciencia en el } \\
\text { estudio de partículas e interacciones? }\end{array}$ & 5 \\
\hline $\begin{array}{l}\text { 13.- ¿Aparece alguna mención a la existencia de otras partículas } \\
\text { 14.- ¿Aparece alguna limitación de los modelos clásicos que tenga que } \\
\text { ser explicada con modelos actualizados? }\end{array}$ & 1 \\
\hline
\end{tabular}


hasta finales del siglo XIX o principios del XX. Después, la pregunta 1, sobre la mención a las interacciones fundamentales, es la que más respuestas positivas ha obtenido, con 7 de los 11 libros haciendo mención. Como detallaremos a continuación, esta mención no aparece en general sistematizada. Por su parte, las preguntas que han obtenido un peor resultado son aquellas que hacen referencia a conceptos de nueva física que no tienen una relación directa con los ítems del currículum, éstas son, las preguntas sobre aceleradores y colisiones (preguntas 7-11), que ponen de manifiesto no sólo si el libro habla de contenidos actualizados sino también si los relaciona con la tecnología y la sociedad (CTS). La pregunta 14 obtiene también muy malos resultados, poniendo de manifiesto que, se hable o no de física moderna, no se hace de una manera sistematizada introduciendo los nuevos conceptos como parte del temario y como respuesta a los límites de los antiguos modelos.

La Figura 1 resume el porcentaje de respuestas positivas a las preguntas de los diferentes libros de texto.

Solamente McGrawHill y Santillana 09 obtienen más de un $50 \%$ de respuestas positivas, pero de manera muy ajustada: $57 \%$ y $50 \%$ respectivamente. El resto de libros no llegan al 50\%, y algunos, como Akal, SM, Ecir 08, Ecir 02 y Oxford, tienen resultados muy negativos con menos de un $20 \%$ de respuestas positivas.

A continuación discutimos los aspectos más relevantes de cada pregunta encontrados en los libros de texto.

\section{Figura 1. Porcentaje de respuestas positivas a las preguntas del cuestionario para libros de los diferentes libros de texto analizados}

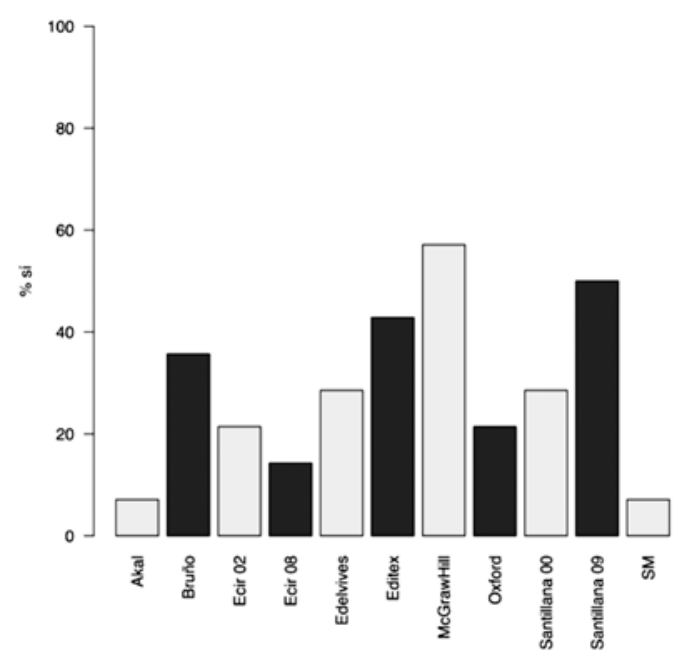

1.- ¿Aparece alguna mención sobre la existencia de cuatro interacciones fundamentales?

Como hemos dicho en el resumen de los resultados, 7 de los 11 libros mencionan las cuatro fuerzas fundamentales; lo cual es un buen resultado. Hay que matizarlo, ya que ninguno de ellos lo hace de una manera sistematizada, es decir, como parte natural del temario, en respuesta a límites clásicos o a través de una estrategia de modelización. Esta mención aparece siempre como un apartado aislado que no se integra en el resto del tema de dinámica. Por ejemplo, Santillana 00 habla de las cuatro fuerzas pero no dice que son fundamentales; sin embargo en Santillana 09 sí las menciona como tal en el tema de dinámica y 
en uno posterior de fuerzas, donde antes de introducirlas, especifica que a partir de las fundamentales se pueden derivar todas las demás. Editex diferencia las fuerzas de contacto (rozamiento, elástica, normal y tensiones) de las fuerzas a distancia (gravitatoria, electromagnética, fuerte y débil), pero no dice que unas son fundamentales y las otras no; $y$ tampoco aprovecha para decir que unas pueden explicarse a partir de las otras. Edelvives habla de las fuerzas fundamentales a través de un texto de Isaac Asimov desde el que se comentan otros aspectos de física moderna; no aparecen en ningún otro apartado del libro. La introducción a través de un texto de divulgación puede ser interesante si luego las conclusiones se integran en el temario; en este caso, la presentación de estos conceptos a través de un texto de divulgación sirve únicamente para mencionarlos de manera marginal y como ideas que aparentemente "están de más" en el tema. Por tanto, aunque sí se habla en general de interacciones fundamentales y se mencionan las cuatro, su introducción es completamente ad-hoc, de manera que más que ayudar a esclarecer ideas, probablemente añaden confusión sobre el resto del temario.

2.- ¿Se explica con ellas el comportamiento de la materia macroscópica?

En cuatro de los casos anteriores en que sí se mencionan las fuerzas fundamentales (Editex, Oxford, Bruño, Edelvives) no se utilizan luego para explicar el comportamiento de la materia macroscópica. Solamente se hace en 4 libros de los analizados, sobre todo cuan- do se habla de la fuerza de rozamiento, en algunos casos también de la elástica, ambas de origen electromagnético. Es curioso el caso de Ecir 08, que aún sin introducir las fuerzas fundamentales dice que "cuando dos cuerpos sólidos están en contacto, se establece entre ellos un conjunto de interacciones, de origen electromagnético, que impiden que penetren los unos en los otros. Estas acciones se producen a nivel atómico y las agrupamos en lo que denominamos fuerzas de contacto." Es importante que los libros establezcan una jerarquía en el listado de fuerzas que introducen, y que no dejen cabos sueltos a la hora de relacionarlas; llama la atención la confusión que existe muchas veces entre el peso y la fuerza gravitatoria, cuando son completamente equivalentes; o la aparente disonancia entre las fuerzas a nivel microscópico (la eléctrica por ejemplo) y sus representaciones macroscópicas, como el rozamiento o la fuerza normal. De ahí, surgen otras muchas confusiones, como identificar la normal como una fuerza de reacción al peso, y no a las fuerzas de apoyo de un cuerpo sobre otro.

3.- ¿Aparece alguna mención a la necesidad de la interacción fuerte para explicar la estabilidad del núcleo?

En los libros en que sí se menciona esta cuestión (Tabla 2) no se explica nada al respecto; simplemente se habla de la existencia de la interacción fuerte y se aprovecha para decir que gracias a ella se entiende que la estabilidad del núcleo, pero ni se cuestiona por qué el núcleo habría de ser inestable ni se rela- 
ciona esta reflexión con ningún otro concepto del tema. Sólo en uno de los casos analizados (Santillana) se ponen los modelos clásicos al límite formulando la pregunta de cómo es posible los protones estén tan cerca en el núcleo atómico si según la interacción eléctrica tendrían que repelerse. Por tanto, para esta pregunta, aunque se han contabilizado 5 respuestas como positivas, a efectos de garantizar un aprendizaje significativo sería más correcto contar 1 .

4.- ¿Aparece alguna mención a la necesidad de las interacciones débiles para explicar la radioactividad?

Los mismos libros que hacían mención a la necesidad de la interacción fuerte para explicar la estabilidad del núcleo, hacen mención ahora de la débil para explicar la radioactividad (Tabla 2), a excepción de Santillana, que en ese caso no dice nada. Esta mención aparece además de la misma manera, como un comentario que ni se explica ni se relaciona con otros conceptos, ni se contextualiza ni hay ninguna referencia histórica al respecto.

5.- ¿Aparece alguna mención al fotón no sólo como cuanto del campo electromagnético, sino como mediador de esta interacción?

En la mayoría de los libros aparece mencionado el fotón cuando se discute el átomo de Bohr y se habla de "cuanto de energía", pero sólo en dos casos aparece explícitamente que el fotón es el mediador de la interacción electromagnética (Tabla 2): Edelvives y McGrawHill. En el primer caso, se menciona a través del texto de divulgación de Asimov, por tanto trasladamos los comentarios que hemos hecho al respecto en la pregunta 1 a esta pregunta. En el segundo, se menciona en un pequeño recuadro separado del hilo argumental del tema. Por tanto, tanto en un caso como en el otro, el tratamiento no es sistemático.

Por otra parte, en el caso de Santillana 09, en el tema de dinámica, se habla de "partícula mensajera" dentro del apartado "fuerzas como interacciones", pero no se dice nada del fotón. Santillana 00 habla de "radiación electromagnética" cuando discute el átomo de Bohr, pero no llega a mencionar siquiera al fotón.

6. - ¿Aparece alguna mención a la interacción como intercambio de partículas?

Los dos libros que hacían mención del fotón como mediador de la interacción electromagnética, mencionan también el concepto de interacciones como intercambio de partículas en el mismo sitio, en el caso de Edelvives en el texto de Asimov, y en el caso de McGrawHill en el mismo recuadro a parte. Por tanto, las conclusiones son similares. Santillana 09 , tal y como hemos dicho en la pregunta 5 , menciona muy de pasada la existencia de "partículas mensajeras", pero no explica cuáles son estas partículas ni habla de las propiedades de las interacciones según sus características.

7.- ¿Aparece alguna mención a los aceleradores de partículas?

8.- ¿Aparece alguna mención sobre el papel de las colisiones en el estudio de las partículas?

9.- ¿Aparece alguna mención sobre el papel de los principios de conservación 
estudiados ( $p, E, q)$ en el estudio de las particulas?

10.- ¿Aparece alguna mención sobre el papel de los campos eléctricos y magnéticos en el funcionamiento del acelerador?

11.- ¿Aparece alguna mención sobre las relaciones CTS en el estudio de partículas e interacciones?

Dados los resultados negativos que ha obtenido este bloque de preguntas, haremos los comentarios al respecto conjuntamente. Todas estas cuestiones hacen referencia a la relación de los conceptos de la física moderna con la tecnología y la sociedad; tanto al impacto que ha tenido la investigación en este campo para el desarrollo social y tecnológico, como al papel de la tecnología en el descubrimiento de nuevas partículas $y$, en general, en el funcionamiento de los aceleradores y colisionadores.

Editex menciona los aceleradores de partículas en el tema del átomo, en un apartado llamado "ciencia y sociedad" que hay al final de todos los temas de este libro. Santillana 00, por su parte, comenta la importancia de la conservación de la energía y el momento lineal en los procesos de choque y desintegración de las partículas, aunque no hace ningún cálculo. El resto de respuestas de todos los libros a este bloque de preguntas es negativo. Por una parte, era de esperar; si ya los conceptos de física moderna necesariamente relacionados con los ítems del currículum no aparecen de manera sistemática, aquellos que lo hacen vía las relaciones CTS, todavía menos, dado el tratamiento que se le da a estas cuestiones en general en los libros de texto. Aunque en este caso, precisamente las relaciones CTS podrían suponer una vía más sencilla y atractiva para introducirlos. Sin embargo, aunque este resultado pudiera ser esperable, llama la atención la clamorosa desactualización de los libros de texto, que se evidencia más que en ninguna otra pregunta del cuestionario, en los resultados de este bloque. Las consecuencias negativas que esto puede tener para el alumnado son de muchos tipos, desde favorecer su desinterés por la asignatura, hasta contribuir a la discusión de ideas obsoletas o crear confusión por la introducción de ciertos conceptos relacionados con otros que no se mencionan. Contribuyen sin duda alguna a la descontextualización de la física y la química en la sociedad e imposibilitan que los estudiantes se hagan una idea remota de cómo se desarrolla en realidad la actividad científica.

12.- ¿Aparece alguna utilización de la historia de la ciencia en el estudio de partículas e interacciones?

Esta pregunta, como hemos dicho antes, ha obtenido respuestas positivas por parte de todos los libros analizados. Queda lejos sin embargo de utilizarse el recurso histórico como parte de la estrategia de modelización, ya que en todos los casos se dan nombres y hechos sin hacer una descripción realista de lo que supuso la investigación del fenómeno correspondiente en el momento. Efectivamente, tanto en los modelos atómicos y, por tanto, en los descubrimientos del protón, electrón y neutrón, así como en el caso de las interacciones eléctrica y gravitatoria, se dan datos históricos. En 
concreto, Editex menciona de pasada la historia de las partículas elementales en un texto a parte del tema. En los otros casos, para ninguna otra de las interacciones ni para otras partículas que no sean el electrón, el protón y el neutrón, en el caso de que se mencionen, se utiliza la historia como un recurso.

13.- ¿Aparece alguna mención a la existencia de otras partículas además del protón, el neutrón y el electrón?

Santillana 09, en el tema de electricidad, al hablar de carga positiva menciona al protón y dice que "hoy en día sabemos que está compuesto por quarks"; a parte de esto, no menciona ninguna partícula más, ni siquiera en el tema del átomo. Oxford habla de quarks y leptones en un párrafo introductorio al tema del átomo. Editex, en el mismo recuadro en que menciona de pasada la historia de las partículas elementales, habla de los quarks y de otras partículas. McGrawHill habla de bosones intermediarios en el mismo sitio en que habla de interacciones como partículas intermediarias; también, en "contenido de ampliación" (unas notas que se encuentran sólo en libro del profesor), menciona otras partículas elementales, incluso el bosón de Higos [sic]. Como vemos, la mención es dispar y completamente a elección de los autores; en ningún caso de manera sistematizada dentro del tema de estructura de la materia.

14.- ¿Aparece alguna limitación de los modelos clásicos que tenga que ser explicada con modelos de partículas e interacciones?
Esta pregunta ha obtenido muy malos resultados, únicamente Santillana 00 hace una reflexión al respecto (pregunta 3), aunque no responde, no llega a explicar exactamente en qué consiste exactamente el papel de la interacción fuerte en la estabilidad del núcleo atómico. Como hemos dicho en los criterios de evaluación, los nuevos conceptos pueden aparecer de una manera o de otra; con esta pregunta se ha pretendido analizar si éstos aparecen, dentro de la estrategia de modelización, como respuesta constructiva a los límites de los modelos anteriores. Vemos que en la práctica totalidad de los casos analizados esto no es así.

Este análisis de textos, se ha completado con entrevistas a profesores de secundaria y bachillerato, siguiendo el guión de preguntas indicado en el apartado de Métodos.

Respecto a la primera pregunta, sobre si se enseña algún concepto actualizado en la línea de la física de partículas, en general la respuesta es que no, y de hacerse, se hace de una manera no sistematizada, sólo a modo de mención. En algún caso, se habla del accidente de Fukushima (CTS) para comentar temas relacionados con la energía nuclear (aunque el profesor no especificó qué se dijo exactamente) o alguna charla sobre el Higgs por el reciente descubrimiento en los experimentos del LHC.

Respecto a la segunda pregunta sobre lo que se hace en la Física de $2^{\circ}$ de bachillerato, la mayoría han señalado que no se trata, aunque algunos profesores han dicho no saberlo por no impar- 
tir la asignatura en este curso. Algunos otros han afirmado directamente que no se habla de física de partículas por culpa de las PAU, aunque un profesor en particular ha incidido en el hecho de que las PAU no suponen ningún condicionante y que segundo sería el "lugar natural" para desarrollar este tipo de conceptos. Algunos profesores han hecho referencia también a que, a pesar de aparecer como un apartado específico en el currículum de segundo en la Comunidad Valenciana, este apartado es el último, su tratamiento es muy superficial y las preguntas que aparecen en las PAU sobre el tema son muy mecanizadas y se limitan a la conservación del número atómico y másico en reacciones nucleares o a desintegraciones radiactivas.

Respecto a la tercera pregunta sobre qué piensan que se podría impartir al respecto y en qué cursos, hay diversas opiniones. Por un lado, hay profesores que han manifestado un problema de “diseño" en el currículum, en el que "no caben" estos temas. Otros, se han mostrado más "integradores" pero siempre desde asignaturas optativas o tipo Ciencias del Mundo Contemporáneo (CMC), o como "cultura general" dentro de la ESO; en ningún caso como parte que impregna toda la asignatura de Física y Química de primero de bachillerato. Sólo en algún caso se ha dicho que sí se podría enseñar, al menos desde la perspectiva CTS, algún concepto más sistematizado. Esta última respuesta ha sido la minoritaria y, en cualquier caso, relativamente vaga.
Finalmente, respecto a las mejoras que este tema puede aportar en cuanto a la comprensión de la naturaleza de la ciencia, las relaciones CTS o el interés de los alumnos por la asignatura; la mayoría han hecho referencia a que estos temas siempre interesan mucho a los estudiantes y sin duda su enseñanza propiciaría un aumento del interés sobre la asignatura. Llama la atención el comentario de un profesor sobre la mejora en la comprensión de la naturaleza de la ciencia, que puntualiza que lo que mejoraría es la comprensión del funcionamiento de la ciencia moderna, refiriéndose a las grandes colaboraciones y a los experimentos caros, en contraposición a cómo funcionaba "antiguamente". En cuanto a las relaciones CTS, algunos mencionan la importancia de conocer las aplicaciones en medicina, y sólo en algún caso del desarrollo de la tecnología en general.

En todas estas reflexiones, pensamos que también influye el factor de formación en contenidos del profesor. En algunos casos, se ha detectado que las ideas sobre cuáles son estos conceptos actualizados respecto a estructura e interacciones de la materia están desordenadas y poco claras, de manera que la primera impresión es que hay cierta reticencia por parte del profesor a tratar este tema.

\section{Conclusiones y perspectivas}

Tras la revisión de 11 libros de texto y de las entrevistas con algunos profesores, podemos afirmar, respecto a las hipótesis enunciadas que, en general, la 
presentación que hacen los libros de los conceptos relacionados con estructura e interacciones de la materia se limita a la de los modelos clásicos. Aparecen ciertas menciones a conceptos actualizados en la línea de la física de partículas, pero de manera no sistematizada y muy dispersa a lo largo del temario, sin establecer relación alguna entre los conceptos, y casi en todos los casos sólo a modo de mención y no de discusión vía la estrategia de modelización. No se hace referencia en general a las relaciones CTS de la física de partículas ni se aprovecha ningún ítem del temario para hablar de estos avances en contexto. La historia de la ciencia se presenta sólo hasta principios del siglo XX, esto ocurre tanto en los modelos atómicos como con la gravedad y la fuerza eléctrica; omitiendo los grandes avances de los últimos 70-80 años. Además, pese a haberse analizado libros de distintos años a lo largo de una una década (a partir del 2000) no se ha encontrado ninguna tendencia que indique una mejoría.

Confirmando lo anterior encontramos que los profesores, pese a ser conscientes de la importancia de este tema, tienen cierta reticencia a tratarlo, posiblemente debido a la falta de formación. Aluden a defectos del currículum o a la complicación de los contenidos modernos para justificar que no se traten de manera sistematizada en la asignatura. En general, proponen que se impartan en otras asignaturas; optativas, como cultura general dentro de otros cursos inferiores, CMC, charlas o a través de relaciones CTS. Así pues, mayoritariamente confirman que no se tratan estos contenidos, ni en primero ni en segundo de bachillerato; y sí creen que sería importante incluirlos en el temario, sea de la manera que sea, de cara a mejorar el interés de los alumnos por la asignatura.

La renovación e inclusión de contenidos actualizados en las asignaturas de Física y Física y Química es esencial para un proceso efectivo de enseñanza-aprendizaje. Se hace por tanto necesario el desarrollo de estrategias y recursos didácticos que permitan transportar al aula estos conceptos. En esta línea, hemos elaborado una propuesta didáctica basada en la Modelización y Personificación (embodiment) (Solbes y Tuzón, 2014) tras un estudio detallado de los problemas que plantea la enseñanza de estos conceptos a estudiantes de Física y Química de primero de bachillerato (Tuzón, 2014).

Como hemos discutido en la introducción, la modelización en el proceso de enseñanza-aprendizaje de las ciencias experimentales, sobre todo dentro del marco socio-constructivista, es una estrategia clave para garantizar un aprendizaje significativo. Es un proceso constructivo en el que las nuevas ideas aparecen sólo a partir de la discusión de los conflictos que generan las ideas previas, a la luz de los resultados, la comunicación con otros investigadores y el contexto. Dentro de esta estrategia, en nuestra propuesta hacemos uso de un proceso de personificación, en el que los alumnos son los agentes activos del modelo que proponen; es decir, utilizan su propio cuerpo para represen- 
tar elementos del modelo e investigan sus límites y propiedades a través de la interacción física con otros alumnos/elementos. La eficacia de esta herramienta en el aprendizaje ha sido demostrada en campos diversos como la neurociencia, la psicología cognitiva, las matemáticas o la física (Johnson-Glenberg, Lindgren, et al., 2012) y se basa en la activación de un gran número de neuronas sensomotoras de manera congruente con el concepto que se esté aprendiendo (JohnsonGlenberg, Lindgren, et al., 2012), dando lugar a un aprendizaje mucho más profundo que desde la mera observación. Además, la interacción con el grupo es un valor añadido, sobre todo de cara a la aparición de propiedades emergentes. Se trata por tanto de un proceso de "personificación y colaboración”.

El objetivo de nuestra propuesta es introducir el nuevo modelo nuclear según el panorama actual de la física de partículas a partir de las concepciones clásicas. Este proceso de modelización está dirigido a estudiantes de bachillerato dentro de las asignaturas de Física y Química o Física. Mediante la personificación de los distintos componentes atómicos y sus interacciones, los estudiantes elaboran el nuevo modelo a distintos niveles de complejidad (átomo, núcleo, partículas elementales) (Solbes y Tuzón, 2014).

La intervención comienza con la identificación de los ingredientes conocidos, relativos a los modelos clásicos, y sus propiedades, que serán personificados por los estudiantes. Estos se dividen en grupos, electrones, neutrones y proto- nes, y se mueven por la sala atendiendo a las consignas de la interacción eléctrica: se repelen si se encuentran con una partícula de su misma carga, se atraen si se encuentran con una partícula de distinta carga o pasan inadvertidos si se encuentran con una partícula neutra. Tras estos ejercicios se discute la escenificación incidiendo en los aspectos más relevantes y se les propone que construyan un átomo utilizando dos de esos protones. Fijar el número de protones permite propiciar discusiones respecto al número de electrones y neutrones que en consecuencia ha de tener un átomo y, de ahí, otras características de los modelos clásicos. La intervención sigue poniendo al límite estos modelos, preguntándoles, por ejemplo, por el papel de los neutrones en el átomo que han construido o por qué los protones, pese a estar tan cerca, no se repelen. Es importante discutir estos aspectos mientras los alumnos escenifican, para que las modificaciones que surjan puedan ser implementadas simultáneamente por los "actores" mismos. Tras distintas respuestas tentativas, se recurre al contexto histórico para completar la discusión. Se aporta un texto y un vídeo sobre el descubrimiento, a principios del siglo XX, de nuevas partículas (lambda, pi, delta, sigma, etc.) a partir de los rayos cósmicos, que invita a los alumnos a pensar en los quarks como componentes de este nueva proliferación de partículas. Con los quarks como nuevas partículas fundamentales, se vuelve a la escenificación del átomo y se discute el nuevo modelo atómico. Cada protón está representado ahora por tres alum- 
nos (quarks) que se cogen de las manos (interacción fuerte); la única manera de que dos protones no se repelan con este modelo, es que los quarks de un protón sientan también la interacción fuerte con los quarks del otro protón, es decir, los alumnos se cogen instantáneamente de las manos de los quarks del protón vecino, representando este efecto con carácter residual. Se discuten después relaciones CTS, como el papel de las colisiones en la detección de partículas o la verosimilitud de las noticas que hablaban del "peligro" del LHC, sabiendo de la existencia de los rayos cósmicos.

La intervención continúa con el mismo esquema, donde la personificación de los modelos, el contexto histórico y las relaciones CTS se retroalimentan y dirigen la discusión. Se introduce la interacción débil y el neutrino como nueva partícula fundamental. Con todas las interacciones fundamentales sobre la mesa, se discute el concepto de "partícula intermediaria"; los alumnos vuelven a escenificar y a reflexionar sobre los alcances de las distintas fuerzas y otras propiedades haciendo representaciones guiadas. Se completa la intervención con conceptos como los de antimateria y sus aplicaciones, la creación de partículas y el bosón de Higgs. Con todo, los alumnos alcanzan una comprensión actualizada sobre la estructura e interacciones de la materia construida por ellos mismos y de la que se analizan, también, las nuevas perspectivas que este nuevo paradigma abre. En este sentido, la aportación fundamental de nuestro estudio es, por una parte, contribuir al diagnóstico de la enseñanza en física de partículas en de los libros de texto, y por otra, proponer un tratamiento con una intervención didáctica que hace uso de la modelización y de una herramienta potente como es la personificación.

\section{Bibliografía}

ACHER, A., ARCÀ, M. y SANMARTÍ, N. (2007). Modeling as a teaching learning process for understanding materials: A case study in primary education. Science Education, $\mathrm{n}^{\circ}$ 91(3), 398-418. DOI: 10.1002/ sce. 20196

ARONS, A.B. (1994). A Guide to Introductory Physics Teaching. John Wiley y Sons.

BOE 147 (2008). Orden ESD/1729.

CUPPARI,A.,RINAUDO, G., ROBUTTI, O. y VIOLINO, P. (1997). Gradual introduction of some aspects of quantum mechanics in a high school curriculum. Physics Education, $\mathrm{n}^{0}$ 32(5), 302-308. DOI: 10.1088/0031$9120 / 32 / 5 / 012$

DOCV 5806 (2008). Decret 102/2008.

VAN DRIEL, J.H.yVERLOOP,N.(2002). Experienced teachers' knowledge of teaching and learning of models and modelling in science education. International Journal of Science Education, n 24(12), 1255-1272. DOI: $10.1080 / 09500690210126711$

FISCHLER, H. y LICHTFELDT, M. (1992). Modern physics and students' conceptions. International Journal of 
Science Education, n ${ }^{\circ}$ 14(2), 181-190. DOI: 10.1080/0950069920140206

FRITZSCH, H. (1984). Los quarks, la materia prima de nuestro Universo. Madrid: Alianza.

GELL-MANN, M. (1995). El quark y el jaguar. Barcelona: Tusquets.

GIL, D., SENENT PÉREZ, F. y SOLBES, J. (1987). La introducción a la física moderna: un ejemplo paradigmático de cambio conceptual. Enseñanza de las Ciencias: revista de investigación y experiencias didácticas, $\mathrm{n}^{0}$ Extra, 209-210.

GIL, D. y SOLBES, J. (1993). The introduction of modern physics: overcoming a deformed vision of science. International Journal of Science Education, $\mathrm{n}^{\circ}$ 15(3), 255-260. DOI: 10.1080/0950069930150303

JOHNSON-GLENBERG, M.C., LINDGREN, R., KOZIUPA, T., BOLLING, A., NAGENDRAN, A., BIRCHFIELD, D. y CRUSE, J. (2012). Serious Games in Embodied Mixed Reality Learning Environments. Proceedings of the Games, Learning and Society Conference, $\mathrm{n}^{0}$ 8.0, 8.

KALKANIS, G., HADZIDAKI, P. y STAVROU,D.(2003).Aninstructional model for a radical conceptual change towards quantum mechanics concepts. Science Education, $\mathrm{n}^{\circ}$ 87(2), 257-280. DOI: 10.1002/sce.10033

KRAGH, H. (2007). Generaciones cuánticas: una historia de la física en el siglo XX. Madrid: Tres Cantos.
LEDERMAN, L. y TERESI, D. (1996). La partícula divina. Barcelona: Crítica.

MOREIRA, M.A. y OSTERMANN, F. (2000). Física contemporánea en la escuela secundaria: una experiencia en el aula involucrando formación de profesores. Enseñanza de las ciencias: revista de investigación y experiencias didácticas, $\mathrm{n}^{0}$ 18(3), 391-404. http:// ddd.uab.cat/record/1501? In=es

NATIONAL RESEARCH COUNCIL (1996). National Science Education Standards. Washington DC: National Academy.

OSTERMANN, F. y CAVALCANTI, C.J.H. (1999). Física Moderna e Contemporânea no ensino médio: elaboração de material didático, em forma de pôster, sobre partículas elementares e interações fundamentais. Caderno Brasileiro de Ensino de Física, ${ }^{0}$ 16(3), 267-286.

PÉREZ, H. y SOLBES, J. (2006). Una propuesta sobre enseñanza de la relatividad en el bachillerato como motivación para el aprendizaje de la física. Enseñanza de las Ciencias: revista de investigación y experiencias didácticas, $\mathrm{n}^{0}$ 24(2), 269-285. DOI:http://ddd.uab.cat/record/21907

PETRI, J. y NIEDDERER, H. (1998). A learning pathway in high-school level quantum atomic physics. International Journal of Science Education, $\mathrm{n}^{0}$ 20(9), 1075-1088. DOI: 10.1080/0950069980200905 
SÁNCHEZ RON, J.M. (1992). El poder de la ciencia: historia socio-económica de la física (siglo XX). Madrid: Alianza.

SÁNCHEZ RON, J.M. (2006). El poder de la ciencia. Madrid: Crítica.

SINARCAS, V. y SOLBES, J. (2013). Dificultades en el aprendizaje y la enseñanza de la Física Cuántica en el bachillerato. Enseñanza de las Ciencias: revista de investigación y experiencias didácticas, $\mathrm{n}^{0} 31(3)$, 9-25. DOI:

http://ddd.uab.cat/pub/edlc/edlc a2013v31n3/edlc a2013v31n3p9.pdf

SOLBES, J. (1996). La física moderna y su enseñanza. Alambique: Didáctica de las ciencias experimentales, $\mathrm{n}^{0} 10$, 59-67.

SOLBES, J. (2009). Dificultades de aprendizaje y cambio conceptual, procedimental y axiológico (I): Resumen del camino avanzado. Revista Eureka sobre Enseñanza y Divulgación de las Ciencias, n 6(1), 2-20. DOI:

http://venus.uca.es/eureka/revista/Volumen $6 / N_{\text {Numero }} 6 \quad 1 /$ Solbes_2009a.pdf

SOLBES, J., CALATAYUD, M., CLIMENT, J. y NAVARRO, J. (1987a). Diseño de un currículum para la introducción del modelo atómico cuántico. Enseñanza de las Ciencias: revista de investigación y experiencias didácticas, $\mathrm{n}^{0}$ extra, 309-310.
SOLBES, J., CALATAYUD, M., CLIMENT, J. y NAVARRO, J. (1987b). Errores conceptuales en los modelos atómicos cuánticos. Enseñanza de las Ciencias: revista de investigación y experiencias didácticas, $\mathrm{n}^{0}$ 5(3), 189-195. DOI: http://ddd.uab. cat/record $/ 43330$

SOLBES, J., SILVESTRE, V. y FURIÓ, C. (2010). El desarrollo histórico de los modelos de átomo y enlace químico y sus implicaciones didácticas. Didáctica de las Ciencias Experimentales y Sociales, $\mathrm{n}^{0} 24,83-105$. DOI: http://ojs.uv.es/index.php/dces/ article/view/2402

SOLBES, J. y TUZÓN, P. (2014). Indagación y modelización del núcleo atómico y sus interacciones. Alambique: Didáctica de las Ciencias Experimentales, $\mathrm{n}^{0} 78$, (en prensa).

STEFANEL, A. (1999). Una experiencia en el marco de la introducción de la física cuántica en la escuela secundaria. Revista de Enseñanza de la Física, $\mathrm{n}^{0}$ 11(2), 35-44.

TIPLER, P.A. (1985). Física moderna. Barcelona: Reverté.

TUZÓN, P. (2014). Diagnóstico del proceso de enseñanza-aprendizaje de la física de partículas en bachillerato. Tesis de Máster. Universitat de València

VELASCO, J. (2000). La textura del món. Alzira: Universitat de ValènciaBromera. 
